01

\title{
Математическое моделирование процесса переноса тепла в эллиптическом канале под действием градиента давления
}

\author{
(C) О.В. Гермидер, В.Н. Попов, А.А. Юшканов \\ Северный (Арктический) фредеральный университет им. М.В. Ломоносова, \\ 163002 Архангельск, Россия \\ e-mail: v.popov@narfu.ru
}

(Поступило в Редакцию 21 марта 2016 г. В окончательной редакции 12 сентября 2016 г.)

\begin{abstract}
Предложен метод расчета потока тепла через поперечное сечение эллиптического канала при наличии параллельного его оси симметрии градиента давления. В качестве основного уравнения, описывающего кинетику процесса, использовано уравнение Вильямса, а в качестве граничного условия на стенке канала модель диффузного отражения. Отклонение состояния газа от равновесного полагается малым. Получены значения потока тепла через поперечное сечение канала в зависимости от значений числа Кнудсена. Проведен сравнительный анализ значений потока тепла при предельном переходе к свободномолекулярному и гидродинамическому режимам.
\end{abstract}

DOI: 10.21883/JTF.2017.03.44234.1813

\section{Введение}

К числу важнейших в прикладном значении задач динамики разреженного газа относится задача о течении разреженного газа в каналах $[1,2]$. Ее строгое теоретическое решение в промежуточной области значений чисел Кнудсена, где неприменимы концепции сплошной среды или свободномолекулярного режима, должно получаться в результате интегрирования уравнения Больцмана (или системы уравнений Больцмана, если газ состоит из молекул разной природы) при соответствующих граничных и начальных условиях [2]. Учитывая сложность кинетического уравнения Больцмана, решение задачи для слаборазреженных газов в общем случае может быть получено с использованием численных методов [1-3]. В последнее время в связи с развитием микро- и наноразмерных технологий более пристальное внимание направлено на кинетический анализ течений газа в каналах различной конфигурации поперечного сечения. В работах $[4,5]$ рассматривалось течение разреженного газа в прямоугольном канале, в [6] - в канале треугольного сечения, в [7-10] — в цилиндрическом канале, в [11] между двумя концентрическими цилиндрами, в [12] - в канале эллиптического сечения. В работах $[4,6-9,11,12]$ используются уравнения с постоянной частотой столкновений. Однако предположение о независимости частоты столкновений молекул газа от их скорости представляет собой достаточно сильное упрощение [13]. Более реалистичным является предположение о постоянстве распределения длины свободного пробега молекул газа по крайней мере для молекул, взаимодействие которых между собой можно аппроксимировать моделью твердых сфер. Это предположение эквивалентно тому, что частота столкновений молекул должна быть пропорциональна абсолютной величине их тепловой скорости. Полученные в $[4,6,8,11,12]$ результаты относятся к задачам, связанным с переносом массы газа в каналах при нали- чии параллельного стенкам канала градиента давления. Вместе с тем представляет интерес и исследование переноса тепла в каналах произвольного сечения. Для цилиндрических каналов в [5,7,10] получены выражения потока тепла при наличии продольного градиента температуры. В [7] задача решена методом дискретных ординат с использованием модели Шахова, а в [10] путем сведения уравнения Вильямса к однородному дифференциальному уравнению первого порядка в частных производных, решение которого получено методом характеристик. Проведенное в [10] сравнение показало, что полученные в этой работе результаты отличаются от аналогичных результатов [7] не более чем на $1 \%$ для режима течения, близкого к свободномолекулярному. Целью настоящей работы является вычисление потока тепла разреженного газа при наличии продольного градиента давления в канале эллиптического сечения. В качестве основного уравнения, описывающего кинетику процесса, в работе использовано уравнение Вильямса, а в качестве граничного условия - модель диффузного отражения.

\section{1. Вывод основных уравнений. Построение функции распределения}

Рассмотрим канал, поперечное сечение которого представляет собой эллипс с полуосями $a^{\prime}$ и $b^{\prime}$. Предположим, что в канале поддерживается постоянный градиент давления, направленный вдоль его оси. Направим оси $O x^{\prime}$ и $O y^{\prime}$ декартовой системы координат вдоль полуосей эллипса, а $O z^{\prime}$ - противоположно градиенту давления. В выбранной системе координат уравнение Вильямса и граничное условие на стенке канала запи- 
сываются в виде [14]

$$
\begin{gathered}
v_{x} \frac{\partial f}{\partial x^{\prime}}+v_{y} \frac{\partial f}{\partial y^{\prime}}+v_{z} \frac{\partial f}{\partial z^{\prime}}=\frac{\omega}{\gamma l_{g}}\left(f_{*}-f\right), \\
f^{+}\left(\mathbf{r}_{s}^{\prime}, \mathbf{v}\right)=f_{s}\left(\mathbf{r}_{s}^{\prime}, \mathbf{v}\right), \quad \mathbf{n v}>0
\end{gathered}
$$

где $\omega=\left|\mathbf{v}-\mathbf{u}\left(\mathbf{r}^{\prime}\right)\right|, \mathbf{v}-$ скорость молекул газа, $\mathbf{u}\left(\mathbf{r}^{\prime}\right)-$ массовая скорость газа, $\mathbf{r}^{\prime}$ - размерный радиус-вектор, $l_{g}=\eta_{g} \beta^{-1 / 2} / p-$ средняя длина свободного пробега молекул газа, $p$ и $\eta_{g}$ - давление и коэффициент динамической вязкости газа, $\gamma=15 / 8, \beta=m /\left(2 k_{B} T_{0}\right)$, $m$ - масса молекулы газа, $k_{B}$ - постоянная Больцмана, $T_{0}$ - температура газа в некоторой точке, принятой в качестве начала координат, $f^{+}\left(\mathbf{r}_{s}^{\prime}, \mathbf{v}\right)$ - функция распределения молекул газа, отраженных от стенки канала, $f_{s}\left(\mathbf{r}_{s}^{\prime}, \mathbf{v}\right)$ - локально-равновесная функция распределения с параметрами, заданными на стенке, $\mathbf{n}$ - вектор нормали к стенке, направленный внутрь канала:

$$
\begin{gathered}
f_{*}=n_{*}\left(\frac{m}{2 \pi k_{B} T_{*}}\right)^{3 / 2} \exp \left(-\frac{m}{2 k_{B} T_{*}}\left(\mathbf{v}-\mathbf{u}_{*}\right)^{2}\right), \\
f_{s}\left(\mathbf{r}_{s}^{\prime}, \mathbf{v}\right)=n(z)\left(\frac{m}{2 \pi k_{B} T_{0}}\right)^{3 / 2} \exp \left(-\frac{m}{2 k_{B} T_{0}} \mathbf{v}^{2}\right) .
\end{gathered}
$$

Параметры $n_{*}, \mathbf{u}_{*}$ и $T_{*}$ в функции $f_{*}$ не являются локальными плотностью, температурой и скоростью и подбираются из условия, что модельный интеграл столкновений удовлетворяет законам сохранения числа частиц, импульса и энергии [13]:

$$
\int \omega M_{j} f_{*} d^{3} \mathbf{v}=\int \omega M_{j} f d^{3} \mathbf{v}, \quad j=0-4,
$$

где $M_{0}=1, M_{i}=m \mathbf{v}_{i}(i=1-3), M_{4}=m v^{2} / 2$.

Будем полагать изменение давления на длине свободного пробега молекул газа малым. В этом случае решение задачи можно получить в линеаризованном виде. Функции $f$ и $f_{*}$ линеаризуем относительно локально равновесной функции распределения

$$
\begin{gathered}
f\left(\mathbf{r}^{\prime}, \mathbf{v}\right)=f_{s}\left(\mathbf{r}_{s}^{\prime}, \mathbf{v}\right)(1+h(\mathbf{r}, \mathbf{C})), \\
f_{*}\left(\mathbf{r}^{\prime}, \mathbf{v}\right)=f_{s}\left(\mathbf{r}_{s}^{\prime}, \mathbf{v}\right)\left(1+h_{*}(\mathbf{r}, \mathbf{C})\right), \\
h_{*}(\mathbf{r}, \mathbf{C})=\frac{\delta n_{*}}{n_{0}}+2 \mathbf{C} \mathbf{U}_{*}+\left(C^{2}-\frac{3}{2}\right) \frac{\delta T_{*}}{T_{0}} .
\end{gathered}
$$

Здесь $\mathbf{C}=\beta^{1 / 2} \mathbf{v}-$ безразмерная скорость молекул газа, $\mathbf{U}_{*}=\beta^{1 / 2} \mathbf{u}_{*}, n_{0}-$ концентрация молекул газа в начале координат, $\mathbf{r}=\mathbf{r}^{\prime} / b^{\prime}-$ безразмерный радиус-вектор. Подставляя (5) и (6) в уравнение (1) и переходя к безразмерным координатам, приходим к уравнению для определения функции $h(\mathbf{r}, \mathbf{C})$

$$
\begin{aligned}
\left(C_{x} \frac{\partial h}{\partial x}+C_{y} \frac{\partial h}{\partial y}\right. & \left.+C_{z} G_{n}(1+h(\mathbf{r}, \mathbf{C}))\right) \\
& \times \gamma \mathrm{Kn}+C h(\mathbf{r}, \mathbf{C})=C h_{*}(\mathbf{r}, \mathbf{C}),
\end{aligned}
$$

где $\mathrm{Kn}=l_{g} / b^{\prime}$ - число Кнудсена, определяющее разреженность газа в канале, $G_{n}-$ безразмерный градиент концентрации молекул газа.

Подставляя выражения (5) и (6) в (4), находим

$$
\begin{aligned}
\frac{\delta n_{*}}{n_{0}}= & \frac{3}{4 \pi} \int C \exp \left(-C^{2}\right) h d^{3} \mathbf{C} \\
& -\frac{1}{8 \pi} \int C^{3} \exp \left(-C^{2}\right) h d^{3} \mathbf{C}, \\
\frac{\delta T_{*}}{T_{0}}= & \frac{1}{2 \pi} \int C \exp \left(-C^{2}\right) h d^{3} \mathbf{C} \\
& -\frac{1}{4 \pi} \int C^{3} \exp \left(-C^{2}\right) h d^{3} \mathbf{C}, \\
U_{*}= & \frac{3}{8 \pi} \int C \mathbf{C} \exp \left(-C^{2}\right) h d^{3} \mathbf{C} .
\end{aligned}
$$

Таким образом, уравнение (6) записывается в виде

$$
\begin{aligned}
& \left(C_{x} \frac{\partial h}{\partial x}+C_{y} \frac{\partial h}{\partial y}+C_{z} G_{n}(1+h(\mathbf{r}, \mathbf{C}))\right) \gamma \mathrm{Kn} \\
& +C h(\mathbf{r}, \mathbf{C})=\frac{C}{2 \pi} \int C^{\prime} \exp \left(-C^{\prime 2}\right) k\left(\mathbf{C}, \mathbf{C}^{\prime}\right) h\left(\mathbf{r}, \mathbf{C}^{\prime}\right) d^{3} \mathbf{C}^{\prime},
\end{aligned}
$$

где $k\left(\mathbf{C}, \mathbf{C}^{\prime}\right)=1+3 \mathbf{C} \mathbf{C}^{\prime} / 2+\left(C^{2}-2\right)\left(C^{\prime 2}-2\right) / 2$.

Решение уравнения (8) ищем в виде

$$
h(\mathbf{r}, \mathbf{C})=G_{n} \operatorname{Kn} \gamma C_{z} Z_{0}\left(x, y, C_{x}, C_{y}\right) .
$$

Здесь $G_{n}-$ безразмерный градиент концентрации молекул газа. В линейном приближении $p(z)=$ $=p_{0}\left(1+G_{p} z\right)$, где $G_{p}=\frac{b^{\prime} d p}{p_{0} d z^{\prime}}-$ безразмерный градиент давления молекул газа. Тогда в предположении постоянства температуры из равенства $p(z)=n(z) k_{B} T_{0}$ в линейном приближении находим $n(z)=n_{0}\left(1+G_{p} z\right)$, т. е. $G_{n}=G_{p}$. С учетом сказанного выражение (9) перепишем в виде

$$
h(\mathbf{r}, \mathbf{C})=G_{p} \operatorname{Kn} \gamma C_{z} Z_{0}\left(x, y, C_{x}, C_{y}\right) .
$$

Подставляя (10) в (8), приходим к уравнению относительно $Z_{0}\left(x, y, C_{x}, C_{y}\right)$ :

$$
\begin{gathered}
\gamma \operatorname{Kn}\left(C_{x} \frac{\partial Z_{0}}{\partial x}+C_{y} \frac{\partial Z_{0}}{\partial y}\right)+C Z_{0}\left(x, y, C_{x}, C_{y}\right)+1 \\
=\frac{3 C}{4 \pi} \int C^{\prime} \exp \left(-C^{\prime 2}\right) C^{\prime 2} Z_{0}\left(x, y, C_{x}^{\prime}, C_{y}^{\prime}\right) d^{3} \mathbf{C}^{\prime}
\end{gathered}
$$

с граничным условием

$$
\left.Z_{0}\left(x, y, C_{x}, C_{y}\right)\right|_{s}=0, \quad \mathbf{n C}>0 .
$$

Для нахождения решения уравнения (12) образуем на множестве функций, зависящих от модуля молекулярной скорости, скалярное произведение с весом $g(C)=C^{5} \exp \left(-C^{2}\right)$ :

$$
\left(f_{1}, f_{2}\right)=\int_{0}^{+\infty} g(C) f_{1}(C) f_{2}(C) d C .
$$


Выберем две ортогональные функции $e_{1}(C)=1$ и $e_{2}(C)=\frac{1}{C}-\frac{3 \sqrt{\pi}}{8}$ (ортогональность понимается здесь как равенство нулю введенного выше скалярного произведения) и разложим $Z_{0}\left(x, y, C_{x}, C_{y}\right)$ по выбранным ортогональным функциям:

$$
\begin{aligned}
Z_{0}\left(x, y, C_{x}, C_{y}\right)= & Z_{1}(x, y, \varphi, \theta) \\
& +\left(\frac{1}{C}-\frac{3 \sqrt{\pi}}{8}\right) Z_{2}(x, y, \varphi, \theta) .
\end{aligned}
$$

При записи (13) мы перешли в пространстве скоростей к сферической системе координат $C_{x}=$ $=C \sin \theta \cos \varphi, C_{y}=C \sin \theta \sin \varphi, C_{z}=C \cos \theta$, где углы $\theta$ и $\varphi$ отсчитываются от положительных направлений осей $C_{z}$ и $C_{x}$. Введем обозначения $c_{x}=C_{x} / C$, $c_{y}=C_{y} / C$.

Подставляя разложение (13) в (11), в силу ортогональности функций $e_{1}(C)$ и $e_{2}(C)$ приходим к системе уравнений

$$
\begin{array}{r}
\left(c_{x} \frac{\partial Z_{1}}{\partial x}+c_{y} \frac{\partial Z_{1}}{\partial y}\right) \gamma \mathrm{Kn}+Z_{1}(x, y, \varphi, \theta)+\frac{3 \sqrt{\pi}}{8} \\
=\frac{3}{4 \pi} \int_{0}^{\pi} \cos ^{2} \theta^{\prime} \sin \theta^{\prime} d \theta^{\prime} \int_{0}^{2 \pi} Z_{1}\left(x, y, \varphi^{\prime}, \theta^{\prime}\right) d \varphi^{\prime}, \\
\left(c_{x} \frac{\partial Z_{2}}{\partial x}+c_{y} \frac{\partial Z_{2}}{\partial y}\right) \gamma \mathrm{Kn}+Z_{2}(x, y, \varphi, \theta)+1=0,
\end{array}
$$

с граничными условиями

$$
\begin{aligned}
& \left.Z_{1}(x, y, \varphi, \theta)\right|_{s}=0, \quad \mathbf{n C}>0, \\
& \left.Z_{2}(x, y, \varphi, \theta)\right|_{s}=0, \quad \mathbf{n C}>0 .
\end{aligned}
$$

Исходя из статистического смысла функции распределения, отличная от нуля компонента вектора потока тепла определяется выражением [3]

$$
\begin{aligned}
q_{z}^{\prime}\left(x^{\prime}, y^{\prime}\right)= & \frac{m}{2} \int\left(v_{z}-u_{z}\left(x^{\prime}, y^{\prime}\right)\right)\left|\mathbf{v}-\mathbf{u}\left(x^{\prime}, y^{\prime}\right)\right|^{2} \\
& \times f\left(\mathbf{r}^{\prime}, \mathbf{v}\right) d^{3} \mathbf{v}=\frac{p_{0}}{\beta^{1 / 2}} q_{z}(x, y),
\end{aligned}
$$

где $q_{z}(x, y)$ - безразмерная компонента вектора потока тепла

$$
\begin{aligned}
& q_{z}(x, y)=\frac{1}{\pi^{3 / 2}} \int \exp \left(-C^{2}\right) C_{z}\left(C^{2}-\frac{5}{2}\right) h(\mathbf{r}, \mathbf{C}) d^{3} \mathbf{C} \\
& =-\frac{G_{p} \gamma \mathrm{Kn}}{4 \pi^{1 / 2}} \int_{0}^{\pi} \cos ^{2} \theta \sin \theta d \theta \int_{0}^{2 \pi} Z_{2}(x, y, \varphi, \theta) d \varphi .
\end{aligned}
$$

Из (16) следует, что функция $Z_{1}(x, y, \varphi, \theta)$ не вносит вклада в вектор потока тепла. Следовательно, решение задачи сводится к отысканию функции $Z_{2}(x, y, \varphi, \theta)$ из уравнения (14) с граничным условием (15).
Решение уравнения (14) с граничным условием (15) находим методом характеристик [15]. Система уравнений характеристик для (14) и два первых ее независимых интеграла имеют вид

$$
\begin{aligned}
\frac{d x}{\gamma \mathrm{Kn} \cos \varphi \sin \theta} & =\frac{d y}{\gamma \mathrm{Kn} \sin \varphi \sin \theta} \\
& =-\frac{d Z_{2}}{Z_{2}(x, y, \varphi, \theta)+1}=d t, \\
y & -x \operatorname{tg} \varphi=C_{1}, \\
\left(Z_{2}(x, y, \varphi, \theta)+1\right) & \exp \left(\frac{x}{\gamma \mathrm{Kn} \cos \varphi \sin \theta}\right)=C_{2} .
\end{aligned}
$$

Исключая из (17) с помощью (15) постоянные интегрирования $C_{1}$ и $C_{2}$, получаем

$$
\begin{aligned}
Z_{2}(x, y, \varphi, \theta)= & \exp \left(-\frac{x}{B_{1}(\varphi, \theta)}\right. \\
& \left.+\frac{B_{3}(x, y, \varphi) \pm B_{4}(x, y, \varphi)}{B_{1}(\varphi, \theta) B_{2}(\varphi)}\right)-1,
\end{aligned}
$$

где верхний знак „, ${ }^{“}$ имеет место при $\frac{\pi}{2} \leq \varphi \leq \frac{3 \pi}{2}$, а нижний знак - при $-\frac{\pi}{2} \leq \varphi \leq \frac{\pi}{2}, a=a^{\prime} / b^{\prime}$,

$$
\begin{gathered}
B_{1}(\varphi, \theta)=\gamma \mathrm{Kn} \sin \theta \cos \varphi, \quad B_{2}(\varphi)=a^{2} \operatorname{tg}^{2} \varphi+1, \\
B_{3}(x, y, \varphi)=-a^{2}(y-x \operatorname{tg} \varphi) \operatorname{tg} \varphi, \\
B_{4}(x, y, \varphi)=a \sqrt{B_{2}(\varphi)-(y-x \operatorname{tg} \varphi)^{2}} .
\end{gathered}
$$

Таким образом, функция распределения молекул газа построена.

\section{2. Вычисление потока тепла в канале. Анализ полученных результатов}

Подставляя $\quad$ построенную функцию $Z_{2}(x, y, \varphi, \theta)$ в (13), находим отличную от нуля компоненту вектора потока тепла согласно (16):

$$
\begin{aligned}
q_{z}(x, y)= & \frac{G_{p} \gamma \mathrm{Kn}}{3 \pi^{1 / 2}}\left(1-\frac{3}{4 \pi} \int_{0}^{\pi}\left(W_{1}(x, y, \theta)\right.\right. \\
+ & \left.\left.W_{2}(x, y, \theta) \cos ^{2} \theta \sin \theta d \theta\right)\right), \\
W_{1}(x, y, \theta)= & \int_{-\pi / 2}^{\pi / 2} \exp \left(-\frac{x}{B_{1}(\varphi, \theta)}\right. \\
& \left.+\frac{B_{3}(x, y, \varphi)-B_{4}(x, y, \varphi)}{B_{1}(\varphi, \theta) B_{2}(\varphi)}\right) d \varphi,
\end{aligned}
$$


Значения $J_{Q} / G_{p}$ при различных значениях $a$

\begin{tabular}{c|c|c|c|c|c}
\hline \multirow{2}{*}{ Kn } & \multicolumn{5}{|c}{$a=a^{\prime} / b^{\prime}$} \\
\cline { 2 - 6 } & 1 & 1.01 & 1.1 & 5 & 10.0 \\
\hline 0.0001 & 0.0001 & 0.0001 & 0.0001 & 0.0001 & 0.0001 \\
0.001 & 0.0007 & 0.0007 & 0.0007 & 0.0007 & 0.0007 \\
0.01 & 0.0070 & 0.0070 & 0.0070 & 0.0070 & 0.0070 \\
0.1 & 0.0656 & 0.0656 & 0.0658 & 0.0672 & 0.0673 \\
0.5 & 0.2434 & 0.2438 & 0.2473 & 0.2763 & 0.2789 \\
1 & 0.3597 & 0.3607 & 0.3685 & 0.4479 & 0.4569 \\
2 & 0.4730 & 0.4746 & 0.4882 & 0.6544 & 0.6809 \\
5 & 0.5912 & 0.5936 & 0.6144 & 0.9268 & 1.0040 \\
10 & 0.6519 & 0.6547 & 0.6796 & 1.0963 & 1.2281 \\
100 & 0.7354 & 0.7390 & 0.7701 & 1.3765 & 1.6519 \\
1000 & 0.7499 & 0.7536 & 0.7859 & 1.4342 & 1.7512 \\
10000 & 0.7519 & 0.7557 & 0.7882 & 1.4430 & 1.7673
\end{tabular}

$$
\begin{aligned}
W_{2}(x, y, \theta)= & \int_{\pi / 2}^{3 \pi / 2} \exp \left(-\frac{x}{B_{1}(\varphi, \theta)}\right. \\
& \left.+\frac{B_{3}(x, y, \varphi)+B_{4}(x, y, \varphi)}{B_{1}(\varphi, \theta) B_{2}(\varphi)}\right) d \varphi .
\end{aligned}
$$

Поток тепла в канале $J_{Q}^{\prime}$ равен [1]

$$
J_{Q}^{\prime}=4 \int_{0}^{b^{\prime}} \int_{0}^{a^{\prime} \sqrt{1-y^{\prime 2} / b^{\prime 2}}} q_{z}^{\prime}\left(x^{\prime}, y^{\prime}\right) d y^{\prime} d x^{\prime}=\frac{p_{0}}{2 \beta^{1 / 2}} J_{Q},
$$

где $J_{Q}-$ безразмерный поток тепла через поперечное сечение канала

$$
J_{Q}=\frac{8}{\pi a} \int_{0}^{1} d y \int_{0}^{a \sqrt{1-y^{2}}} q_{z}(x, y) d x .
$$

Приведенный поток тепла, найденный согласно (19), не зависит непосредственно от полуосей эллипса, а определяется их отношением $a=a^{\prime} / b^{\prime}$ и числом Кнудсена Kn. Значения $J_{Q}$, найденные согласно (19) с использованием системы компьютерной алгебры Maple 17 при различных значениях числа Кнудсена и отношениях полуосей эллипса $a^{\prime}$ и $b^{\prime}$, приведены в таблице. В свободномолекулярном режиме для эллиптических каналов с равными полуосями поток тепла через поперечное сечение имеет значение, равное $4 /(3 / \sqrt{\pi})[1]$, которое получаем в пределе при $a=1$ из (19), раскладывая в ряд по малому параметру $1 / \mathrm{Kn}$ подынтегральные выражения в интегралах $W_{1}(x, y, \theta), W_{2}(x, y, \theta)$ и отбрасывая в них члены второго и выше порядка малости.

В гидродинамическом режиме значение приведенного потока тепла через поперечное сечение равно нулю [1]. При $\mathrm{Kn} \ll 1$ значения потока тепла, вычисленные согласно (19), также стремятся к нулю.

\section{Заключение}

В настоящей работе решена задача о вычислении потока тепла в канале эллиптического сечения при наличии постоянного градиента давления, направленного вдоль оси симметрии канала. Получены значения потока тепла через поперечное сечение канала в широких пределах изменения числа Кнудсена. Показано, что в предельных случаях, когда $\mathrm{Kn} \ll 1$ и $\mathrm{Kn} \gg 1$, полученные в работе результаты переходят в аналогичные значения потока тепла для гидродинамического и свободномолекулярного режимов соответственно.

Работа выполнена при частичном финансировании в рамках Государственного задания „Создание вычислительной инфраструктуры для решения наукоемких прикладных задач““ (проект № 3628).

\section{Список литературы}

[1] Шарипов Ф.М., Селезнев В.Д. Движение разреженных газов в каналах и микроканалах. Екатеринбург: УрО РАН, 2008. $230 \mathrm{c}$.

[2] Кошмаров Ю.А., Рыжков Ю.А. Прикладная динамика разреженного газа. М.: Машиностроение, 1977. 184 с.

[3] Коган М.Н. Динамика разреженного газа. Кинетическая теория. М.: Наука, 1967. 440 с.

[4] Tuтарев B.A., Шахов E.M. // Журн. вычисл. матем. и матем. физ. 2010. Т. 50. № 7. С. 1285-1302.

[5] Гермидер О.В., Попов В.Н., Юиканов А.А. // ЖТФ. 2016. Т. 86. Вып. 6. С. 37-41.

[6] Naris S., Valougeorgis D. // Europ. J. Mechan. B. Fluids. 2008. Vol. 27. P. 810-822.

[7] Siewert C.E., Valougeorgis D. // J. Quant. Spectr. Radiativ. Transfer. 2002. Vol. 72. P. 531-550.

[8] Taheri P., Bahrami M. // Phys. Rev. 2012. Vol. 86. P. 1-9.

[9] Kamphorst C.H., Rodrigues P., Barichello L.B. // Appl. Mathemat. 2014. Vol. 5. P. 1516-1527.

[10] Гермидер О.В., Попов В.Н., Юшканов А.А. // Журн. Средневолжского матем. общества. 2015. Т. 17. № 1. C. 22-29.

[11] Шахов Е.М. // Журн. вычисл. матем. и матем. физ. 2003. T. 43. № 7. C. 1107-1116.

[12] Graur I., Sharipov F. // Europ. J. Mechan. B. Fluids. 2008. Vol. 27. P. 335-345.

[13] Черчиньяни К. Математические методы в кинетической теории газов. М.: Мир, 1973. 245 с.

[14] Гулакова С.В., Попов В.Н. // Инжен. физ. журн. 2014. Т. 87. № 4. C. 953-960.

[15] Курант Р. Уравнения с частными производными. М.: Мир, 1964. $830 \mathrm{c}$. 